\title{
Diagnóstico do USO DE TÉCNICAS DE CALAGEM E ADUBAÇÃO DOS ASSENTAMENTOS RURAIS DO ESTADO DE SÃO PAULO: ESTUDO DE CASO
}

\section{Diagnosis the use of lime and fertilization techniques in a agricultural settlement of São Paulo state: case study}

\author{
Uso de técnicas de diagnóstico de cal y fertilización de los \\ asentamientos rurales de São Paulo: un estudio de caso
}

\author{
Michelle Sato Frigo ${ }^{1}$ \\ Osmar de Carvalho Bueno² \\ Elisandro Pires Frigo ${ }^{3}$
}

\begin{abstract}
RESUMO
No contexto do projeto "Desenvolvimento e capacitação de agricultores no uso de técnicas para correção do solo em assentamentos rurais", a identificação do uso de técnicas agrícolas faz-se necessária como uma ferramenta importante para tomadas de decisão, principalmente no tocante às políticas públicas de Assistência Técnica e Extensão Rural (ATER). Desta forma, o presente estudo teve por objetivo diagnosticar o uso de técnicas de calagem e adubação nestes assentamentos rurais de modo a identificar possíveis políticas públicas de ATER. Do total de famílias abordadas, 79,89\% responderam ao questionário elaborado para este diagnóstico. A pesquisa de campo foi realizada através de aplicação de questionário elaborado para este estudo $e$ a análise dos resultados divididos em perfil dos beneficiários, conhecimento de técnicas de manejo de solos e uso agrí́cola das áreas dos Projetos de Assentamento pelos beneficiários. Concluiu-se que embora a grande maioria dos beneficiários declararem-se trabalhadores de área rural e lavradores esta informação não está em coerência com a de conhecimento do tipo de solo, pois este tipo de compreensão da paisagem é primordial para as tomadas de decisão com relação ao uso agrícola do solo, além disso, o acesso ao conhecimento $e$ uso das técnicas de calagem $e$ adubação vem diminuindo nos assentamentos rurais sob vigência do II Plano Nacional de Reforma Agrária, o que entra em total discordância com as premissas do mesmo. Cabe aqui destacar a importância de políticas públicas de ATER dirigidas a estes beneficiários da Reforma Agrária, principalmente com relação ao manejo de solos.
\end{abstract}

Palavras-chave: diagnóstico, Reforma Agrária, II Plano Nacional de Reforma Agrária, calagem, adubação.

\section{SUMMARY}

In the context of the project "Development and qualification of agriculturists in the use of techniques for correction of the ground in agricultural assentament", the identification of the use of agricultural techniques becomes necessary as an important tool for decision taking, mainly in regards to the public politics of Assistance Technique and Agricultural Extension (ATER). In such a way, the present study had for objective to diagnosis the use of lime and fertilization techniques in these agricultural assentament in order to identify possible public politics ATER. Of the total of boarded families, $79.89 \%$ had answered to the question-

\footnotetext{
1 Engenheira Agrônoma. Doutora em Agronomia "Energia na Agricultura", Docente do curso de Agronomia, UFPR, Palotina/PR Brasil. E-mail: msfrigo@ufpr.br.

2 Engenheiro Agrônomo, Prof. Adjunto no Departamento de Gestão e Tecnologia Agroindustrial, campus de Botucatu, Faculdade de Ciências Agronômicas - UNESP. Fazenda Experimental Lageado, Rua José Barbosa de Barros, nº. 1780, Botucatu/SP, CEP: 18.610307. Caixa postal 237, fax: (014) 3815-3438. Brasil. E-mail: osmar@fca.unesp.br.

3 Engenheiro Agrícola, Doutor em Agronomia "Irrigação e Drenagem", Docente do curso de Agronomia, UFPR, Palotina/PR - Brasil. E-mail: epfrigo@ufpr.br.
} 
naire elaborated for this diagnosis. The field research was carried through application of questionnaire elaborated for this study and the analysis of the results divided in profile of the beneficiaries, knowledge of techniques of ground handling and agricultural use of the areas of the Projects of Assentament for the beneficiaries. It was concluded that even so the great majority of the beneficiaries agricultural area and farmers to be declared diligent of this information are not in coherence with the one of knowledge of the type of ground, therefore, this type of understanding of the landscape is primordial for the taking of decision with regard to the agricultural use of the ground, moreover, the access to the knowledge and use of the techniques of lime and fertilization comes diminishing in the agricultural assentament under validity of II National Plan of the Agrarian Reformation, what it enters in total discord with the premises of the same. It fits here to detach the importance of public politics ATER directed these beneficiaries of the Agrarian Reformation, mainly with regard to the ground handling.

Keywords: diagnosis, Agrarian Reformation, II National Plan of the Agrarian Reformation, lime, fertilization.

\section{RESUMEN}

En el marco del proyecto "Desarrollo y formación de los agricultores en el uso de técnicas para la remediación del suelo en asentamientos rurales," la identificación de la utilización de técnicas agrícolas es necesaria como una herramienta importante para la toma de decisiones, especialmente en lo que respecta a las políticas públicas Asistencia técnica y Extensión Rural (ATER). Por lo tanto, el presente estudio tuvo como objetivo diagnosticar el uso de técnicas de encalado y fertilización en estos asentamientos rurales con el fin de identificar posibles políticas ATER pública. De todas las familias se acercaron, 79,89\% respondió al cuestionario preparado para este diagnóstico. La investigación de campo se llevó a cabo a través de un cuestionario elaborado para este estudio y el análisis de los resultados divididos en el perfil de los beneficiarios, los conocimientos técnicos de la gestión del suelo y el uso de las áreas agrícolas de Proyectos de liquidación de los beneficiarios. Se concluyó que, aunque la gran mayoría de los beneficiarios se declaran a los trabajadores rurales y los agricultores que esta información no es consistente con el conocimiento del tipo de suelo, ya que este tipo de comprensión del paisaje es fundamental para la toma de decisiones sobre el uso agrícola de la tierra, por otra parte, el acceso al conocimiento y uso de las técnicas de fertilización y encalado ha disminuido en los asentamientos rurales en el término de la II plan Nacional de Reforma agraria, que entra en total desacuerdo con los supuestos de la misma. Cabe destacar la importancia de las políticas públicas dirigidas a los beneficiarios de la reforma agraria ATER, en particular en relación con la gestión del suelo.

Palabras-clave: Diagnóstico, Reforma Agraria, II Plan Nacional de Reforma Agraria, encalado, fertilización.

\section{Introdução}

A luta pela terra e a necessidade de manutenção e/ou melhoria da qualidade do solo, em seus aspectos químicos, físicos e biológicos, são lados da mesma questão que envolve a reprodução social dos assentados, de modo que estes se fortaleçam.

A reação do solo é o primeiro fator que deve ser levado em conta quando se pretende fazer algum cultivo, isso porque, se ela não for favorável, medidas corretivas deverão ser tomadas antes do plantio e do preparo do solo(adubação).

No Brasil, a maioria dos solos apresenta acidez elevada, ou seja, $\mathrm{pH}$ menor que cinco (SOARES; SILVA; ZAMBALDE, 2003).

Segundo Goedert e Lobato (1988), o conhecimento atual sobre os solos que cobrem o território brasileiro é ainda muito geral, mas é suficiente para concluir que a maioria deles apresenta algum tipo de limitação para a agricultura, especialmente para a agricultura de altos rendimentos. As limitações mais comuns podem ser agrupadas em cinco tipos: I) acidez e baixa fertilidade; II) susceptibilidade à erosão; III) má drenagem; IV) salinidade; e V) textura excessivamente arenosa.

Em termos gerais, as práticas de calagem e adubação visavam transformar a camada arável do solo, e todo o sistema de recomen- 
dação desses insumos estava limitado a essa camada do solo.

Nos últimos anos, com a expansão da agricultura para áreas com solos em estádo avançado de intemperismo e com baixa capacidade de retenção de água, tem-se verificado que é vital considerar também as camadas subsuperficiais do solo no sistema de manejo. Esse aspecto tem sido muito discutido no Brasil, face à complexidade dos fenômenos que o envolvem (GOEDERT; LOBATO,1988)

A calagem é considerada como uma das práticas que mais contribui para o aumento da eficiência dos adubos e consequentemente, da produtividade e da rentabilidade agropecuária.

Apesar deste fato, ela ainda é subutilizada, tendo em vista a pouca informação recebida em nível de campo pelos lavradores.

A adubação é um procedimento realizado para nutrição das plantas, principalmente em plantações comerciais. A definição dos adubos ou fertilizantes a serem utilizados depende de uma composição entre as necessidades da cultura e da oferta de nutrientes do solo em que ela está sendo cultivada. Assim, a recomendação de adubação ou da quantidade e composição dos fertilizantes a serem aplicados dependem diretamente das condições do solo, observada através de uma análise de solo, e da requisição nutricional da cultura para atingir a produtividade desejada (FETT, 2005).

A adubação, segundo Lopes, Silva e Guilherme (1990), começa com a análise de solo, continua com a calagem e termina com a aplicação do adubo adequado.

Assim, partindo da proposição do II Plano Nacional de Reforma Agrária (II PNRA) da inserção da Universidade em políticas públicas, particularmente da implementação de redes de Assistência Técnica e Extensão Rural (ATER), surgiu o convênio entre o Instituto Nacional de Colonização e Reforma Agrária (INCRA), superintendência de São Paulo e Fundação de
Estudos e Pesquisas Agrícolas e Florestais (FE$\mathrm{PAF}$ ), tendo como interveniente a Faculdade de Ciências Agronômicas (FCA) da UNESP (Universidade Estadual de São Paulo) - campus de Botucatu/SP, com o propósito de executar o projeto "Desenvolvimento e capacitação de agricultores no uso de técnicas para correção do solo em assentamentos rurais".

O objetivo do projeto foi disponibilizar calcário aos agricultores familiares assentados em Projetos de Assentamento Rural de responsabilidade do INCRA, no estado de São Paulo. Foram envolvidos inicialmente 53 Projetos de Assentamento em todo o estado, sendo beneficiadas 4.283 famílias, que além de receberem o calcário propriamente dito, também participaram de sensibilização e capacitação acerca da importância do solo e sua correção pela calagem, técnicas de amostragem e coleta de solo para análise.

Neste contexto, a identificação do uso de determinadas técnicas para a definição de sistemas de produção mais adequados, faz-se necessário como uma ferramenta importante para tomadas de decisão, principalmente no tocante às políticas públicas de ATER.

$\mathrm{O}$ conceito de sistema de produção agrícola é muito variado, podendo compreender desde uma lavoura apenas, até todos os componentes do setor primário de um país.

Um sistema de produção pode ser definido como uma combinação (no tempo e no espaço) dos recursos disponíveis para a obtenção das produções vegetais e animais, podendo também ser concebido como uma combinação de diversos subsistemas produtivos de culturas vegetais, animais e de processamento de produtos agrícolas (DUFUMIER, 1996).

Desta forma, o presente estudo teve por objetivo diagnosticar o uso de técnicas de calagem e adubação nos Projetos de Assentamento envolvidos no projeto "Desenvolvimento e capacitação de agricultores no uso de técnicas para correção do solo em assentamentos rurais", 
de modo a identificar possíveis políticas públicas de ATER.

\section{Material e métodos}

Do total de famílias abordadas no projeto, participaram e efetivamente receberam calcário, 76,35\%, sendo que deste total, 79,89\% responderam ao questionário elaborado para este diagnóstico, ou seja, 2.616 famílias em 53 Projetos de Assentamento em todo o estado de São Paulo.

Para tanto, a pesquisa de campo foi através da aplicação de questionário elaborado para este estudo, composto de questões fechadas formadas por blocos de questões: I) Identificação do beneficiário, da área do lote e da força de trabalho de sua família (idade, número de pessoas na produção, atividade rurais em que atuou); II) Conhecimentos acerca do solo e técnicas de calagem $e$ adubação (conhecimento ou não $e$ utilização ou não de tais técnicas, bem como o tempo e o uso de assistência técnica nos mesmos); III) Uso agrícola (conhecimento sobre o uso das áreas anteriormente à formação do Projeto de Assentamento, produção atual, produção futura e técnicas pretendidas no manejo desta).

Para análise dos dados, estes foram agrupados em três grupos distintos de acordo com o tempo de estabelecimento do Projeto e da vigência ou não do II PNRA, sendo desta forma agrupados em: Projetos de Assentamento com mais de 5 anos (estabelecidos e sem a vigência do II PNRA), Projetos de Assentamento com 5 a 2 anos (estabelecidos e com a vigência do II PNRA) e Projetos de Assentamento com menos de 2 anos (recém-estabelecidos e com a vigência do II PNRA).

As informações obtidas através da aplicação do questionário foram submetidas a técnicas de estatística descritiva básica.

\section{Resultados e discussões}

Os Projetos de Assentamento atendidos pelo projeto "Desenvolvimento e capacitação de agricultores no uso de técnicas para correção do solo em assentamentos rurais"

Os assentamentos da reforma agrária são comprovadamente uma luta das famílias assentadas pela construção de um novo modo de vida alicerçado num projeto de desenvolvimento rural. A vida familiar, nesse contexto, é marcada pela da união de seus membros em torno do ideal comum de melhores condições de vida, mas também pela união necessária para o estabelecimento de sistemas agrícolas sustentáveis no longo prazo (BORGES et al., 2004).

Continuando, segundo Borges (2004), a família assentada é o ponto central para a compreensão da vida cotidiana no assentamento, nos aspectos que caracterizam a obtenção de expressões de condições e de qualidade de vida centrada na construção de um modo particular de desenvolvimento: a de assentado.

É nesse cotidiano que ocorrem as mediações entre o vivido, o concebido, e onde o conhecimento empírico acerca de diversas práticas agrícolas é discutido e disseminado. $\mathrm{O}$ ponto central deste diagnóstico foram as famílias assentadas, na figura do chefe de família (geralmente, homem ou mulher).

Todos os Projetos de Assentamento estudados são de responsabilidade federal e são objetos de intervenção e discussão de políticas de ATER, em vários níveis e por vários órgãos, como por exemplo, ITESP (Fundação Instituto de Terras de São Paulo), EMBRAPA (Empresa Brasileira de Pesquisa Agropecuária), Secretarias Municipais de Agricultura, ONGs (Organizações Não-Governamentais), entre outros.

Duas diferentes políticas públicas, porém complementares, estão sendo desenvolvidas nestas áreas: 
- Assentamentos com mais de 5 anos: recuperação destes projetos;

- Assentamentos com 5 a 2 anos e com menos de 2 anos: implantação de novos projetos com modelos adequados a cada bioma local.

Em ambas, o objetivo principal do II PNRA é a qualidade, fator preponderante neste processo, portanto, neste contexto, práticas culturais adequadas, como o manejo correto do solo, fazem-se não somente necessárias, como também fatores importantes no desenvolvimento sustentável destes Projetos de Assentamento.

\section{Análise Descritiva}

\section{Perfil dos beneficiários}

Em todos os grupos analisados a idade dos beneficiários é de 41 a 50 anos, sendo que tanto nos Projetos de Assentamentos com mais de 5 anos e com de 5 a 2 anos, a faixa etária predominante é de 46 a 50 anos, com 14,15\% e $16,24 \%$ respectivamente. Nos Projetos com menos de 2 anos, predominou a faixa etária de 41 a 45 anos, com $15,27 \%$ do total dos beneficiários.

Com relação à força de trabalho que por definição são as pessoas que compõem uma organização e que contribuem para a consecução das suas estratégias, dos seus objetivos e das suas metas, tais como: empregados em tempo integral ou parcial, temporários, autônomos e contratados de terceiros que trabalham sob a coordenação direta da organização; neste caso, a força de trabalho é representada pelos membros da família aptos em idade e condições de saúde ao trabalho agrícola.

Em todos os três grupos analisados a força de trabalho é representada por duas pessoas, representando $29,41 \%$ em Projetos de mais de 5 anos, 32,56\% em Projetos de 5 a 2 anos e $32,67 \%$ em Projetos de menos de 2 anos, conforme pode ser observado na Figura 1.

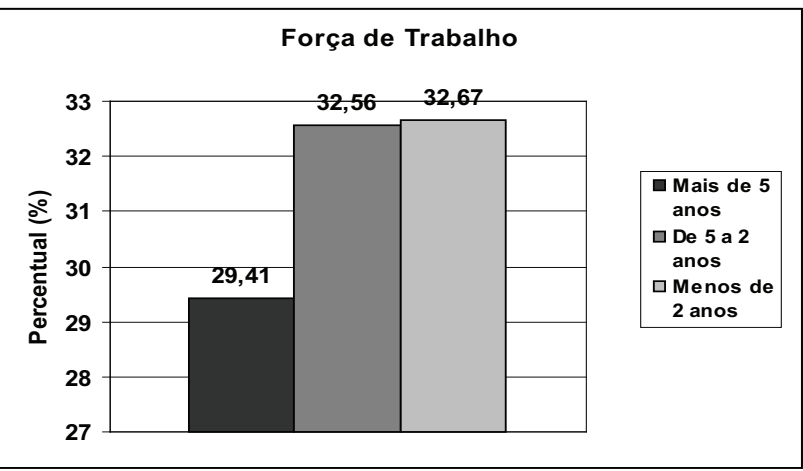

FIGURA 1: FORÇA DE TRABALHO NOS GRUPOS ANALISADOS - DUAS PESSOAS.

FONTE: DADOS DE PESQUISA EM CAMPO (2006).

A grande maioria dos beneficiários declararam-se como trabalhadores rurais, em todos os três grupos analisados, com 98,26\%, $98,13 \%$ e $98,50 \%$, respectivamente nos Projetos de mais de 5 anos, de 5 a 2 anos e de menos de 2 anos (Figura 2).

Da mesma forma, os beneficiários declarantes como ex-lavradores, ou seja, ex-agricultores rurais, em seus trabalhos na área rural, anteriores ao ingresso nos Projetos de Assentamento foram a grande maioria, representados por $81,49 \%$ nos Projetos de mais de 5 anos, 68,05\% nos de 5 a 2 anos e $67,71 \%$ nos de menos de 2 anos (Figura 3).

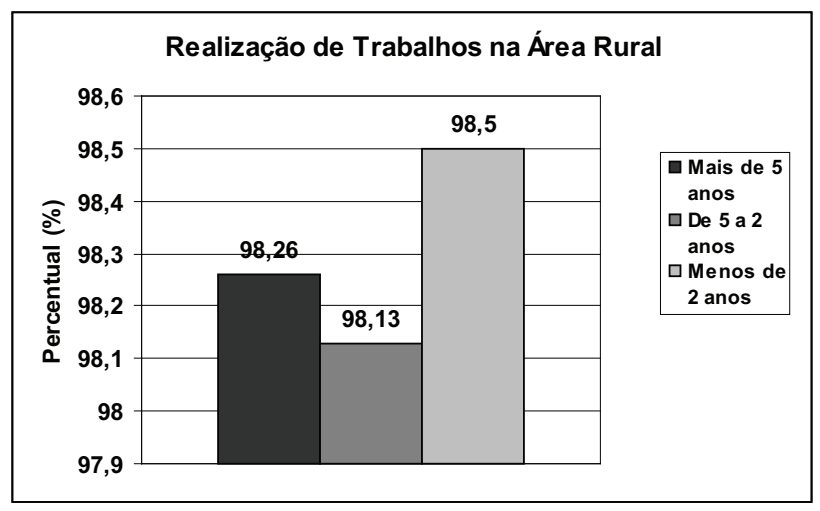

FIGURA 2: REALIZAÇÃO DE TRABALHOS NA ÁREA RURAL PELOS GRUPOS ANALISADOS. FONTE: DADOS DE PESQUISA EM CAMPO (2006). 
Conhecimento de técnicas de manejo de solos: calagem e adubação

A percepção dos beneficiários acerca da paisagem rural onde está inserida sua área é um princípio bastante importante para o desenvolvimento de técnicas agrícolas, principalmente no que diz respeito aos conhecimentos empíricos de manejo.

Em todos os grupos analisados o desconhecimento acerca do tipo de solo (argiloso ou arenoso) de sua área pelo beneficiário é predominante, sendo que este percentual aumenta de grupo para grupo, em sentido crescente, ou seja, nos Projetos de Assentamentos mais antigos o desconhecimento é menor (embora bastante significativo) do que nos mais recentes $(66,85 \%$ nos de mais de 5 anos, $71,10 \%$ nos de 5 a 2 anos e $80,97 \%$ nos de menos de 2 anos, conforme a Figura 4).

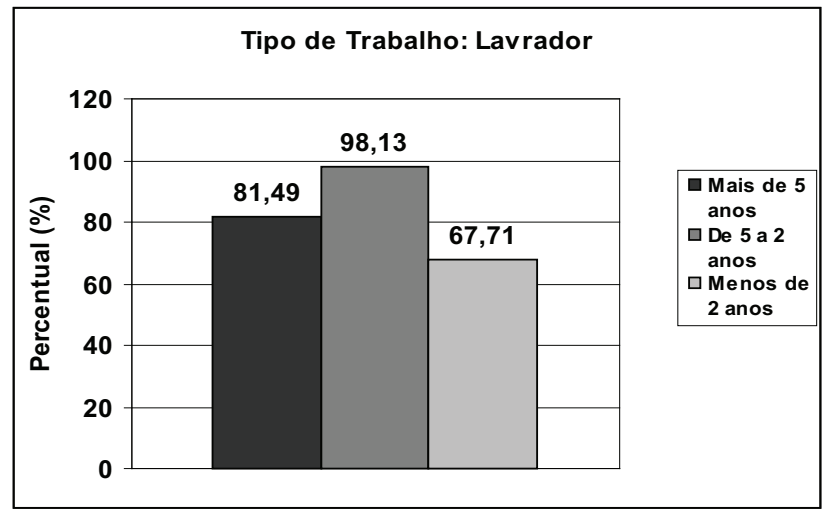

FIGURA 3: TIPO DE TRABALHO REALIZADO ANTERIORMENTE AO INGRESSO NO PROJETO DE ASSENTAMENTO (LAVRADOR).

FONTE: DADOS DE PESQUISA EM CAMPO (2006).

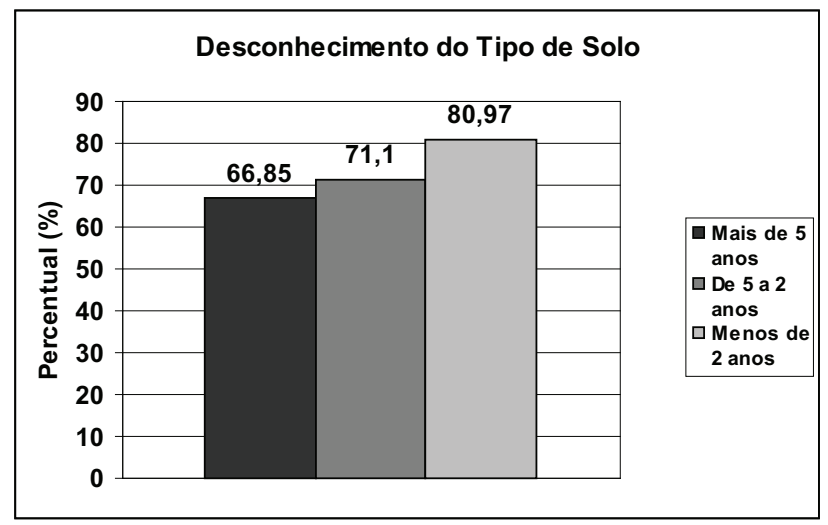

FIGURA 4: DESCONHECIMENTO DO TIPO DE SOLO PELO BENEFICIÁRIO.

FONTE: DADOS DE PESQUISA EM CAMPO (2006).
Da mesma forma que o conhecimento do tipo de solo, comporta-se a realização de análise de solo, além de não ter sido realizada pela grande maioria dos beneficiários, também aumenta de forma crescente dos assentamentos mais antigos para os mais recentes, de 58,98\% em Projetos de mais de 5 anos, 93,99\% nos de 5 a 2 anos e $98,42 \%$ nos de menos de 2 anos (Figura 5).

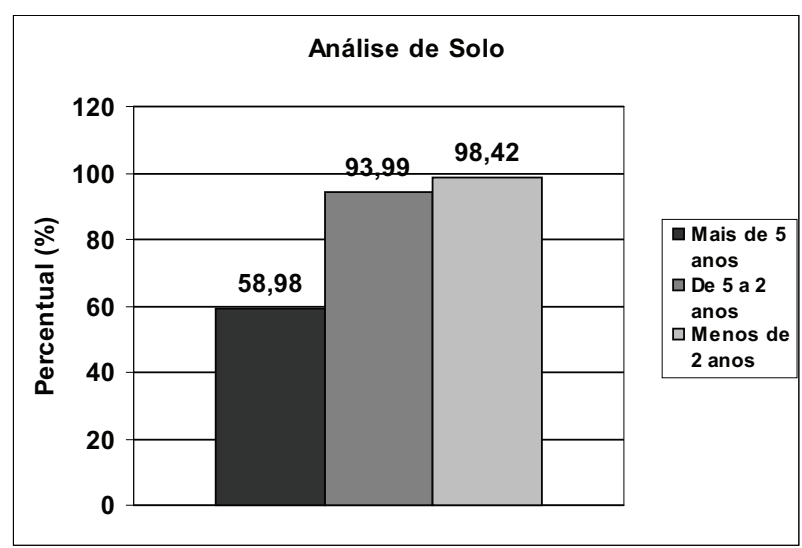

FIGURA 5: NÃO REALIZAÇÃO DE ANÁLISE DE SOLO PELOS PROJETOS DE ASSENTAMENTO ANALISADOS.

FONTE: DADOS DE PESQUISA EM CAMPO, (2006).

Dentre os beneficiários que realizaram análise de solo de suas áreas, os de Projetos de Assentamentos de mais de 5 anos, 43,32\%, realizaram a mais de 5 anos; os de Projetos de 5 a 2 anos, $52,38 \%$, realizaram a 1 ano ou menos e os de menos de 2 anos, 60,00\% realizaram a mais de 5 anos, anteriormente às áreas terem sido desapropriadas, na época de acampamento destas famílias.

A operação de calagem também acompanha o crescimento crescente de não utilização da técnica, assim como observado a respeito do conhecimento do solo pelo beneficiário $e$ da não realização de análise de solo $(63,06 \%$ em Projetos de mais de 5 anos, 85,21\% nos de 5 a 2 anos e 97,76\% nos de menos de 2 anos, conforme a Figura 6). 


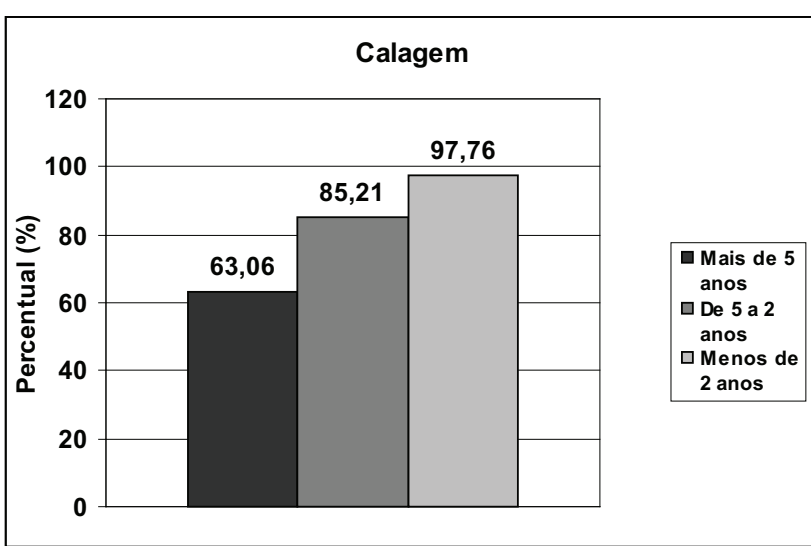

FIGURA 6: NÃO UTILIZAÇÃO DA TÉCNICA DE CALAGEM PELOS BENEFICIÁRIOS DOS PROJETOS DE ASSENTAMENTO ANALISADOS

FONTE: DADOS DE PESQUISA EM CAMPO (2006).

Seguindo a lógica do tempo de realização de análise de solo, nos Projetos de mais de 5 anos, dos que realizaram calagem, $41,87 \%$ realizaram a mais de 5 anos; nos de 5 a 2 anos, $66,67 \%$ a 1 ano ou menos; e nos de menos de 2 anos, 44,44\%, a mais de 5 anos, na época anterior à criação dos Projetos de Assentamento.

Com relação ao acesso de assistência técnica tanto para a realização da análise de solo como para a calagem, dos beneficiários que declararam utilizar as duas técnicas e que não tiveram acesso a assistência técnica, nos Projetos de mais de 5 anos, 34,70\% não tiveram acesso, nos de 5 a 2 anos, 59,57\% e nos de menos de 2 anos, 54,54\%.

Na operação de adubação a não utilização da técnica também foi crescente, sendo que nos Projetos de mais de 5 anos, 49,16\% não utilizaram, nos de 5 a 2 anos, foram $81,87 \%$ e nos de menos de 2 anos, 95,19\% (Figura 7).

Com relação ao tempo da última adubação, todos os grupos analisados e que realizaram adubação, todos fizeram a 1 ano ou menos, sendo $41,45 \%$ para os de mais de 5 anos, $61,40 \%$ nos de 5 a 2 anos e 80,00\% nos de menos de 2 anos.

Dentre estes beneficiários que utilizaram a técnica da adubação, a grande maioria não teve acesso a orientação técnica para esta operação, sendo que nos Projetos de Assentamento de mais de 5 anos, estes beneficiários representaram $51,08 \%$ do total, nos de 5 a 2 anos, 67,86\%, e nos de menos de 2 anos, 71,43\%.

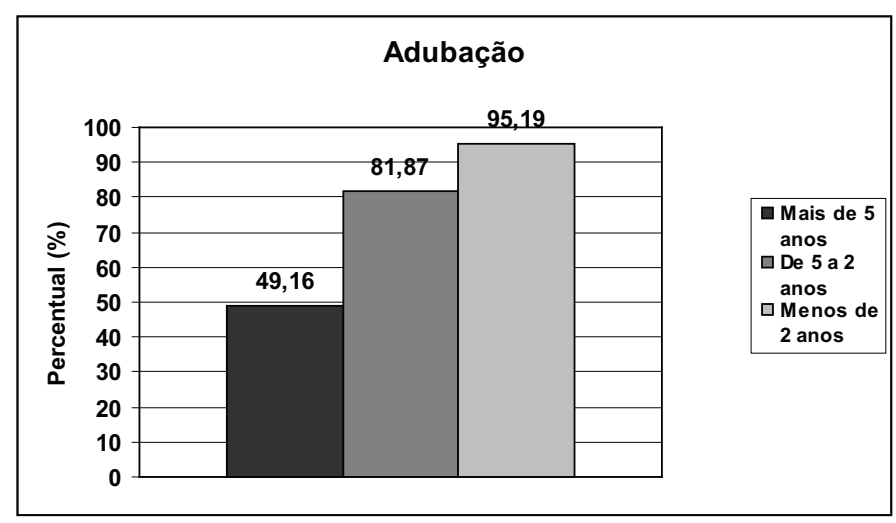

FIGURA 7: NÃO UTILIZAÇÃO DA TÉCNICA DE ADUBAÇÃO PELOS BENEFICIÁRIOS DOS PROJETOS DE ASSENTAMENTO ANALISADOS

FONTE: DADOS DE PESQUISA EM CAMPO (2006).

Uso agrícola das áreas dos Projetos de Assentamento pelos beneficiários

O uso das terras anteriormente à criação dos Projetos de Assentamento, indicam em muitos casos a conservação destas áreas, sendo, portanto, esta informação de grande importância para uma avaliação indireta do estado de conservação destes solos.

Nos projetos de Assentamentos de mais de 5 anos e nos de 5 a 2 anos, anteriormente à criação do Projeto, estas áreas eram pastagens abandonadas em $54,49 \%$ e $87,58 \%$, respectivamente. Já nos Projetos de menos de 2 anos, 49,31\% destas áreas eram lavouras.

Atualmente, a criação de gado de leite é a principal atividade nos Projetos de Assentamento com mais de 5 anos e nos de 5 a 2 anos (28,46\% e $38,68 \%$ respectivamente), sendo que nos de menos de 2 anos, a grande maioria ainda não estava produzindo nada (38,49\%).

A produção futura de milho é o objetivo de $43,59 \%$ dos beneficiários de Projetos de Assentamento de 5 a 2 anos e de 33,76\% dos de menos de 2 anos; os de mais de 5 anos, os que 
ainda não trabalham com gado de leite, 25,15\% pretendem começar a trabalhar.

As técnicas pretendidas de serem utilizadas nestas futuras produções, são de calagem e adubação para os beneficiários de Projetos de Assentamento de mais de 5 anos (32,57\%) e nos de 5 a 2 anos (26,20\%). Nos de menos de 2 anos, a principal técnica pretendida de ser utilizada é a mecanização, com $34,31 \%$.

\section{Conclusões}

Embora a grande maioria dos beneficiários dos Projetos de Assentamento analisados neste estudo declarar-se como trabalhadores de área rural e lavradores (ou agricultores), esta informação não está em coerência com a informação de conhecimento do tipo de solo, pois qualquer trabalhador de área rural e lavrador, no caso deste último mais ainda, tem este tipo de compreensão da paisagem de seu meio de produção, seja por na grande maioria das vezes conhecimentos empíricos ou informações difundidas através da assistência técnica, já que esta informação é primordial para as tomadas de decisão com relação à que tipo de cultura utilizar em determinada área.

$\mathrm{O}$ acesso ao conhecimento $e$ ao uso das técnicas de análise de solo, calagem e adubação, seja por iniciativa própria ou através de assistência técnica, vem diminuindo nos Projetos de Assentamento mais recentes, sob vigência do II PNRA, o que entra em total discordância com as premissas do mesmo, que prevê não somente uma Reforma Agrária massiva, mas com qualidade, sustentável a longo prazo. Para tanto, tais técnicas são fundamentais para a garantia do uso adequado, bem como da conservação dos solos, assim como da melhora da produção $e$ produtividade destas áreas.

Observa-se que por se tratarem de áreas de pastagens abandonadas, os solos das áreas de Projetos de Assentamento com mais de 5 anos e com 5 a 2 anos, são áreas bastante degradadas, o que ficou comprovado conforme Sato et al. (2006), em estudo acerca dos dados preliminares do projeto "Desenvolvimento $e$ capacitação de agricultores no uso de técnicas para correção do solo em assentamentos rurais", em que na média, os solos destas áreas são essencialmente ácidos (na faixa de 4,0-5,5 de pH), necessitando para o bom desenvolvimento das culturas e para a disponibilização adequada de nutrientes pela adubação, de correção através de calagem. Embora os Projetos de menos de 2 anos encontrarem-se em áreas anteriormente com lavouras, estas também possuem solos ácidos, necessitando de correção pela calagem.

A produção atual destas áreas entra em coerência com o estado atual do solo das mesmas, a criação de gado para a produção de leite torna-se uma das únicas alternativas viáveis em solos com tais níveis de degradação, em função de que nelas já existem pastos formados; embora não sejam altamente eficientes é uma fonte de renda e subsistência importante para estas famílias.

Para que futuramente essas áreas possam produzir plenamente, sejam pastagens para gado de leite, ou milho, como declarado por muitos beneficiários, será indispensável o uso de técnicas como a calagem e a adubação, entre outras, bem como uma assistência técnica mais efetiva.

Cabe aqui destacar a importância de políticas públicas de ATER dirigidas aos beneficiários da Reforma Agrária; em primeiro lugar devido à grande parte destes beneficiários possivelmente não terem nenhum ou pouco conhecimento de práticas agrícolas, independentemente de sua origem e, em segundo lugar, devido a estas áreas possuírem solos degradados e com problemas de fertilidade, como já discutido.

Tanto quanto necessária, como possível, a reprodução social destas terras depende destas políticas públicas de ATER, assim como a 
proposta do II PNRA de qualidade no processo de Reforma Agrária.

\section{REFERÊNCIAS}

ANJOS, F. S. et al. Agricultura familiar e políticas públicas: o impacto do PRONAF no Rio Grande do Sul. Revista brasileira de Economia e Sociologia Rural, Brasília, v. 42, n. 3, p. 529-548, 2004.

BARRETO, R. C. S.; KHAN, A. S.; LIMA, P. V. P. S. Sustentabilidade dos assentamentos do município de Caucaia-CE. Revista brasileira de Economia e Sociologia Rural, Brasília, v. 43, n. 2, p. 225-247, 2004.

BORGES, J. R. P.; FABBORO, A. L. D.; RODRIGUEZ JR, A. L. Percepção de riscos socioambientais no uso de agrotóxicos - o caso dos assentados da reforma agrária paulista. In: XIV ENCONTRO NACIONAL DE ESTUDOS POPULACIONAIS, ABEP, 2004, Caxambú. Anais... Caxambú: ABEP, 2004.

DUFUMIER, M. Les projets de développement agricoleManuel d'expertise. Paris: CTA-Karthala, 1996.

FETT, M. S. Serviço Brasileiro de Respostas Técnicas: adubação, novembro de 2005. Rio Grande do Sul: SENAI/ RS - Departamento Regional. Disponível em: < http://sbrt. ibict.br/upload/sbrt1658.pdf > . Acesso em: 25/4/2007.

FEPAF. Projeto "Desenvolvimento e capacitação de agricultores no uso de técnicas para correção do solo em assentamento rurais". Botucatu: INCRA/FEPAF, 2005, 5p. (Projeto 30000/2005 Instituto Nacional de Colonização e Reforma Agrária - Superintendência de São Paulo/ Fundação de Estudos e Pesquisas Agrícolas e Florestais).

INCRA. Instituto Nacional de Colonização e Reforma Agrária. Análise diagnóstico de sistemas agrários: guia metodológico. Disponível em: <http://www.incra.gov. br/arquivos/0143901397.pdf> . Acesso em: 02/4/2007.

LOBATO, E.; GOEDERT, W. J. O Solo como Base dos Sistemas de Produção Agrícola. In: CONGRESSO BRASILEIRO DE CIÊNCIA DO SOLO, 11, 1988, Campinas. Anais... Campinas: 1988, p. 101-108.

LOPES, A. S. ; SILVA, M. C. ; GUILHERME, L. R. G. Acidez do solo e calagem. 3 ed. São Paulo: ANDA, 1990, 22 p.

II PNRA. II Plano Nacional de Reforma Agrária. Disponível em: <http://www.mda.gov.br/arquivos/PNRA 2004.pdf>. Acesso em: 04/4/2007.

SANT'ANA, A. L.; COSTA, V. M. H. M. Produtores familiares e estratégias ligadas à Terra. Revista Brasileira de Economia e Sociologia Rural, Brasília, v. 42, n. 04, p. 663-683, 2004.

SATO, M. et al. Extensão rural e assistência técnica: pressupostos para a Reforma Agrária no século XXI. In: SIMPÓSIO "REFORMA AGRÁRIA: BALANÇO CRÍTICO E PERSPECTIVAS”, 2006, Uberlândia. Anais... UFU, 2006. Disponível em:<http://www.simposioreformaagraria.propp.ufu.br/> . Acesso em 04/4/2007.

SOARES, A. H. V.; SILVA, C. A.; ZAMBALDE, A. L. Um Sistema Especialista para o Cálculo da Necessidade de Calagem e Recomendação de Corretivo. CeresSefs, Lavras, 2003. Disponível em: <http://www.dcc.ufla.br/ infocomp/artigos/v3.2/art08.pdf> . Acesso em 23/4/2007. 